\title{
Fatores influentes na escolha de rota dos ciclistas
}

Isabel-Cristina Nunes de Sousa. Investigadora independente, São Carlos, Brasil. Suely da Penha-Sanches. Universidade Federal de São Carlos, São Carlos, Brasil.

RESUMO | Para determinação dos aspectos mais relevantes no planejamento de redes cicloviárias, um questionário foi respondido por ciclistas em viagens utilitárias (por motivo de trabalho e/ou estudo) na cidade de São José dos Campos-sP. Para o processamento das respostas, análises estatísticas descritivas foram feitas para representação do perfil sociodemográfico dos respondentes, além de posteriores comparações com a amostra de ciclistas da pesquisa Origem-Destino realizada em 2011 na referida cidade. Também foram feitos testes de hipóteses (estatística inferencial) para verificação da existência de diferenças estatisticamente significativas de opinião, em função de características dos perfis identificados, e confrontação dos resultados obtidos com as informaçóes encontradas durante revisão bibliográfica. A prioridade dos ciclistas da amostra é chegar o mais rapidamente possível a seus destinos, por caminhos iluminados e seguros, além de pedalar o minimamente necessário, preferencialmente por vias com pavimento em bom estado de conservação.

PALAVRAS-CHAVE $\mid$ mobilidade, transporte urbano, planejamento urbano.

ABSTRACT | For the determination of the most relevant aspects of Bicycle-Network Planning, a questionnaire regarding utilitarian trips (work andlor study related reasons) was answered by cyclists in the City of São José dos Campos. For the processing of responses, descriptive statistical analyses were conducted to represent sociodemographic profiles and posterior comparisons with the sample of cyclists from the Origin-Destination research held in 2011 for the same city. A hypothesis testing (inferential statistics) was also completed to verify the existence of significant opinion differences, due to the characteristics of the identified profiles, and a confrontation between the final results and the information gathered during the bibliographic review. The priority of the cyclists from the sample is to arrive as soon as possible to their destination through illuminated and safe paths, alongside pedaling the necessary minimum, preferably on streets with good pavement conditions.

KEYWORDS | mobility, urban transportation, urban planning. 


\section{Introduçáo}

A bicicleta é uma opção de transporte urbano mais sustentável que os modos motorizados, em virtude de seus benefícios sociais, ambientais, econômicos e de saúde física e mental (Anderson, Schnohr, Schroll \& Hein, 2000; Bassett, Pucher, Buehler, Thompson \& Crouter, 2008; Dora \& Philips, 2000; Godefrooij, Pardo \& Sagaris, 2009; Gordon-Larsen et al., 2009; Hamer \& Chida, 2008; Hrncir, Song, Zilecky \& Jakob, 2014; Li, Wang, Liu \& Ragland, 2012; Oja et al., 2011; Winters \& Teschke, 2010).

No intuito de transformar o ambiente urbano em um lugar seguro e confortável para pedalar, e tornar o transporte cicloviário um componente integrado da almejada intermodalidade no transporte, são necessários dados sobre as características dos ciclistas e seus comportamentos de viagem (Casello, Rewa \& Nour, 2012).

Uma das informações-chave para a definição de redes cicloviárias funcionais, que atendam aos desejos de deslocamento dos usuários, reside na identificação das rotas utilizadas pelos ciclistas, e as razóes para escolha das mesmas (Segadilha \& Sanches, 2014a).

As pesquisas Origem-Destino $(\mathrm{O} / \mathrm{D})$, se realizadas periodicamente, permitem acompanhar a evoluçáo dos padróes de deslocamento da população e da mobilidade em geral (Baptista et al., 2009). Apesar de serem úteis para a aferiçâo do comportamento de viagem e da demanda de transportes (Raia, 2000, p. 117), as pesquisas O/D não fornecem informaçóes sobre a escolha das rotas percorridas e, devido aos custos e ao planejamento necessário para sua execuçáo, não são empregadas regularmente na maioria das cidades brasileiras (Instituto de Políticas de Transporte e Desenvolvimento [ITDP], 2017).

No planejamento cicloviário, o conhecimento dos determinantes na escolha de rota pelos ciclistas pode facilitar a concepção de instalaçóes cicloviárias (Stinson \& Bhat, 2003), de modo a priorizar as vias mais atraentes para os ciclistas e os percursos mais frequentados (Segadilha \& Sanches, 2014a), além de determinar os condicionantes que podem contribuir para o aumento do uso da bicicleta (Yang \& Mesbah, 2013).

\section{Principais fatores que influenciam na escolha das rotas}

Agrupados por categorias, os estudos consultados durante revisão bibliográfica sobre os fatores que influenciam na escolha do trajeto ciclístico integram a Tabela 1. A maioria dos estudos examinados não engloba a realidade sul americana, tendo sido realizados, predominantemente, em países europeus e norte-americanos. Segadilha (2014) e Pitilin (2016), por outro lado, realizaram estudos no Brasil, elencando diversos fatores considerados importantes para a escolha da rota, identificados por meio de extensa revisão bibliográfica, e servindo de base para esta pesquisa.

Nos estudos supracitados, nota-se uma preferência, entre os ciclistas, por rotas com infraestrutura cicloviária contínua, ausência de estacionamentos para automóveis nas vias, baixos volumes de tráfego, limites reduzidos de velocidade, além de trajetos curtos e rápidos. Ademais, foram constatadas diferenças de comportamento entre ciclistas experientes e inexperientes, regulares e ocasionais, e entre homens e 
mulheres, além da distinção entre o comportamento de ciclistas idosos que são mais cautelosos, e ciclistas jovens, que priorizam a rapidez no deslocamento.

Apesar de existirem divergências quanto à declividade e à sinalização de parada obrigatória nos estudos encontrados, sobressaem-se indicativos sobre a preferência por percursos planos, ou com aclives moderados, e predileçáo pela menor quantidade de sinalizaçóes de parada obrigatória (semáforos, cruzamentos, sinais de PARE, etc.).

Trata-se de contingente expressivo de estudos que apresentam pontos em comum, sendo necessário a complementação com pesquisas no contexto sul americano, de modo a detectar novos fatores além dos elencados na Tabela 1.

Logo, o intuito desta pesquisa é, juntamente ao delineamento do perfil sociodemográfico de ciclistas de uma cidade brasileira de porte médio, identificar os principais fatores que influenciam na escolha da rota por estes usuários, de modo a estabelecer um paralelo com a literatura científica sobre o assunto. Além de efetuar um comparativo da amostra com o universo da pesquisa o/D, realizada em 2011 pelo Instituto de Pesquisa, Administração e Planejamento (IPPLAN) na cidade utilizada como estudo de caso, São José dos Campos-sP.

\section{TABELA I Fatores que influenciam na escolha das rotas pelos ciclistas}

\begin{tabular}{|c|c|}
\hline FATORES & REFERÊNCIAS \\
\hline \multicolumn{2}{|r|}{ CARACTERÍSTICAS FÍSICAS } \\
\hline $\begin{array}{l}\text { Existência de } \\
\text { infraestruturas } \\
\text { cicloviárias }\end{array}$ & $\begin{array}{l}\text { Aultman-Hall, Hall, e Baetz (1997); Abraham, McMillan, Rownlee, e Hunt } \\
\text { (2002); Moudon et al. (2005); Stinson e Bhat (2003, 2004); Krizek (2006); } \\
\text { Hunt e Abraham (2007); Krizek, El-Geneidy e Thompson (2007); Raford, } \\
\text { Chiaradia e Gil (2007) Tilahun, Levinson e Krizek (2007); Bernhoft e Cars- } \\
\text { tensen (2008); Sener, Eluru e Bhat (2009); Dill (2009); Menghini, Carrasco, } \\
\text { Schüssler \& Axhausen (2010); Winters, Teschke, Grant, Setton \& Brauer } \\
\text { (2010); Winters e Teschke (2010); Broach, Gliebe e Dill (2009, 2011); Hood, } \\
\text { Sall e Charlton (2011); Larsen e El-Geneidy (2011); Broach, Dill e Gliebe } \\
\text { (2012); Caulfield, Brick e McCarthy (2012); Li et al. (2012); Kang e Fricker } \\
\text { (2013); Snizek, Nielsen e Skov-Petersen (2013); Krenn, Oja e Titze (2014); } \\
\text { Pitilin e Sanches (2016). }\end{array}$ \\
\hline $\begin{array}{l}\text { Conservação do } \\
\text { pavimento }\end{array}$ & $\begin{array}{l}\text { Antonakos (1994); Aultman-Hall, et al. (1997); Stinson e Bhat (2003, 2004); } \\
\text { Bernhoft e Carstensen (2008); Casello, Nour e Rewa (2011); Winters e Teschke } \\
\text { (2010); Kang e Fricker (2013); Segadilha e Sanches (2014b, 2014c, 2014d); } \\
\text { Pitilin e Sanches (2016). }\end{array}$ \\
\hline $\begin{array}{l}\text { Topografia/Declivi- } \\
\text { dade }\end{array}$ & $\begin{array}{l}\text { Fajans e Curry (2001); Stinson e Bhat (2003, 2004); Sener et al. (2009); Men- } \\
\text { ghini et al. (2010); Winters et al. (2010); Broach et al. (2009, 2011); Hood et } \\
\text { al. (2011); Broach et al. (2012); Li et al. (2012); Milakis, Athanasopoulos, Va- } \\
\text { feiadis, Vasileiadis \& Vlastos (2012); Koh e Wong (2013); Krenn et al. (2014); } \\
\text { Segadilha e Sanches (2014a, 2014b). }\end{array}$ \\
\hline $\begin{array}{l}\text { Estacionamentos para } \\
\text { automóveis }\end{array}$ & $\begin{array}{l}\text { Stinson e Bhat (2003, 2004); Krizek (2006); Tilahun et al. (2007); Bernhoft e } \\
\text { Carstensen (2008); Sener et al. (2009); Winters e Teschke (2010). }\end{array}$ \\
\hline Barreiras/Obstáculos & $\begin{array}{l}\text { Aultman-Hall et al. (1997); Stinson e Bhat (2003); Casello et al. (2012); } \\
\text { Emond e Handy (2012). }\end{array}$ \\
\hline $\begin{array}{l}\text { Largura/Número de } \\
\text { faixas de tráfego }\end{array}$ & 97); Sener et al. (2009). \\
\hline
\end{tabular}


(continuaçâo)

\begin{tabular}{|c|c|}
\hline FATORES & REFERÊNCIAS \\
\hline \multicolumn{2}{|r|}{ CARACTERÍSTICAS DO TRÁFEGO } \\
\hline Sinalizaçōes de parada & $\begin{array}{l}\text { Aultman-Hall et al. (1997); Fajans e Curry (2001); Stinson e Bhat (2003, } \\
\text { 2004); El-Geneidy, Krizek e Iacono (2007); Krizek et al. (2007); Bernhoft e } \\
\text { Carstensen (2008); Sener et al. (2009); Menghini et al. (2010); Broach et al. } \\
\text { (2009, 2011); Broach et al. (2012); Casello et al. (2011); Caulfield et al. (2012); } \\
\text { Snizek et al. (2013); Krenn et al. (2014). }\end{array}$ \\
\hline Limite de velocidade & $\begin{array}{l}\text { Aultman-Hall et al. (1997); El-Geneidy et al. (2007); Sener et al. (2009); } \\
\text { Caulfield et al. (2012); Milakis et al. (2012); Krenn et al. (2014); Segadilha e } \\
\text { Sanches (2014a, 2014b). }\end{array}$ \\
\hline $\begin{array}{l}\text { Volume de veículos } \\
\text { motorizados }\end{array}$ & $\begin{array}{l}\text { Aultman-Hall et al. (1997); Abraham et al. (2002); Stinson e Bhat (2003, } \\
\text { 2004); El-Geneidy et al. (2007); Broach et al. (2009, 2011); Sener et al. (2009); } \\
\text { Casello et al. (2011); Winters et al. (2010); Hood et al. (2011); Broach et al. } \\
\text { (2012); Caulfield et al. (2012); Li et al. (2012); Milakis et al. (2012); Kang e } \\
\text { Fricker (2013); Segadilha e Sanches (2014a, 2014b, 2014c, 2014d); Pitilin e } \\
\text { Sanches (2016). }\end{array}$ \\
\hline $\begin{array}{l}\text { Composição do } \\
\text { tráfego }\end{array}$ & $\begin{array}{l}\text { Sener et al. (2009); Winters et al. (2010); Snizek et al. (2013); Segadilha e } \\
\text { Sanches (2014a, 2014b). }\end{array}$ \\
\hline \multicolumn{2}{|r|}{ CARACTERÍSTICAS AMBIENTAIS } \\
\hline Iluminação & Sener et al. (2009); Segadilha e Sanches (2014b). \\
\hline Arborização & $\begin{array}{l}\text { Winters et al. (2010); Lee, Jennings e El-Geneidy (2011); Snizek et al. (2013); } \\
\text { Krenn et al. (2014). }\end{array}$ \\
\hline Ocupaçáo do entorno & $\begin{array}{l}\text { Stinson e Bhat (2003); Dill (2009); Casello et al. (2011); Lee, Jennings e El- } \\
\text {-Geneidy (2011); Koh e Wong (2013); Krenn et al. (2014). }\end{array}$ \\
\hline $\begin{array}{l}\text { Seguridade (sensação } \\
\text { de segurança pessoal) }\end{array}$ & $\begin{array}{l}\text { Stinson e Bhat (2003); Sener et al. (2009); Casello et al. (2011); Larsen e El- } \\
\text {-Geneidy (2011); Li et al. (2012); Kang e Fricker (2013); Koh e Wong (2013); } \\
\text { Segadilha e Sanches (2014b); Pitilin e Sanches (2016). }\end{array}$ \\
\hline \multicolumn{2}{|r|}{ CARACTERÍSTICAS DA VIAGEM } \\
\hline Tempo de viagem & $\begin{array}{l}\text { Stinson e Bhat (2003, 2004); Krizek (2006); Tilahun et al. (2007); Sener et al. } \\
\text { (2009); Broach et al. (2011); Casello et al. (2011); Hood et al. (2011); Caul- } \\
\text { field et al. (2012); Yang e Mesbah (2013); Hrncir et al. (2014). }\end{array}$ \\
\hline $\begin{array}{l}\text { Comprimento/ } \\
\text { Distância }\end{array}$ & $\begin{array}{l}\text { Aultman-Hall et al. (1997); Abraham et al. (2002); Krizek et al. (2007); Dill } \\
\text { (2009); Broach et al. (2009, 2011); Hood et al. (2011); Broach et al. (2012); } \\
\text { Casello et al. (2012); Menghini et al. (2010); Winters et al. (2010); Casello et } \\
\text { al. (2011); Koh e Wong (2013); Yang e Mesbah (2013); Beheshtitabar, Ríos, } \\
\text { König-Hollerwöger, Svatý e Rydergren (2014); Krenn et al. (2014); Segadilha e } \\
\text { Sanches (2014c, 2014d). }\end{array}$ \\
\hline \multicolumn{2}{|r|}{ CARACTERÍSTICAS DO CICLISTA } \\
\hline Gênero & $\begin{array}{l}\text { Antonakos (1994); Aultman-Hall et al. (1997); Krizek, Johnson e Tilahun } \\
\text { (2004); El-Geneidy et al. (2007); Tilahun et al. (2007); Dill e Gliebe (2008); } \\
\text { Broach et al. (2009); Sener et al. (2009); Bernhoft e Carstensen (2008); Winters } \\
\text { e Teschke (2010); Hood et al. (2011); Kang e Fricker (2013); Segadilha e } \\
\text { Sanches (2014c, 2014d). }\end{array}$ \\
\hline $\begin{array}{l}\text { Frequência de uso/ } \\
\text { Experiência }\end{array}$ & $\begin{array}{l}\text { Antonakos (1994); Stinson e Bhat (2004); El-Geneidy et al. (2007); Hunt e } \\
\text { Abraham (2007); Sener et al. (2009); Winters et al. (2010); Winters e Teschke } \\
\text { (2010); Hood et al. (2011); Caulfield et al. (2012); Larsen e El-Geneidy (2011); } \\
\text { Kang e Fricker (2013). }\end{array}$ \\
\hline Idade & Stinson e Bhat (2003); Bernhoft e Carstensen (2008); Sener et al. (2009). \\
\hline
\end{tabular}

FONTE ELABORAÇÃo PRÓPRIA 


\section{Obtençáo dos dados - opiniáo dos ciclistas}

Visando caracterizar o perfil dos usuários e obter suas opiniôes sobre os fatores influentes na escolha de rota, foi aplicado um questionário entre ciclistas que realizam viagens utilitárias (por motivo de trabalho e/ou estudo) na cidade de São José dos Campos-sp. Os participantes foram encontrados em Polos Geradores de Viagens (PGVs) - locais com potencial de atrair grande quantidade de usuários da bicicleta, como escolas, universidades, indústrias, e terminais de transporte coletivo (Land Transport Safety Authority [LTSA], 2004; Godefrooij, Pardo \& Sagaris, 2009; ITDP, 2011).

Pessoas que já utilizam a bicicleta como modo de transporte, por conhecerem os problemas e peculiaridades das rotas que utilizam, são capazes de apontar os fatores que mais influenciam na escolha de seus trajetos (LTSA, 2004; Godefrooij, Pardo \& Sagaris, 2009). Assim, a seleção da amostra ocorreu pelo método de amostragem não probabilística "bola de neve" (snowball), em que um ciclista, encontrado em um PGV, indica um ou mais ciclistas para participar da pesquisa e compor a amostra. Este tipo de amostragem pode ser utilizado em populaçóes raras, com reduzido número de integrantes (Appolinário, 2012), como é o caso dos usuários da bicicleta na maioria das cidades brasileiras.

\section{Instrumento de pesquisa}

Por ser uma técnica padronizada de levantamento de dados, o questionário facilita a codificação, tabulação e tratamento dos dados obtidos, além de permitir a realização de análises estatísticas. A utilização de categorias diferenciadas, como questóes fechadas, possibilita inserir escalas de resposta - série graduada de itens que medem o grau/intensidade das opinióes e atitudes, e não a qualidade destas. Dentre as escalas de resposta existentes, a de diferencial semântico solicita que a pessoa marque uma posição entre dois adjetivos antagônicos em uma escala bipolar, normalmente de sete ou cinco pontos (Gil, 2008).

Possuindo questôes sobre o perfil sociodemográfico do usuário (motivo da viagem, gênero, idade, frequência e tempo de uso da bicicleta como modo de transporte), e uma questão com escala de resposta de diferencial semântico de 5 pontos, sobre a importância de diversos fatores para a escolha da rota, o questionário elaborado foi testado previamente por meio de uma pesquisa-piloto, realizada no $3^{\circ}$ trimestre de 2015 com 30 ciclistas, de modo a identificar possíveis dificuldades de compreensão nas perguntas formuladas, e verificar se o questionário não estava sendo exaustivo ao respondente (muito longo).

Dentre as modificaçôes feitas após a aplicação preliminar do questionário, destacase a transferência das questóes sobre o perfil sociodemográfico para o começo, de modo a iniciar os questionamentos com perguntas facilmente assimiláveis pelos participantes e, gradualmente, direcionar para questóes de maior complexidade, além da inclusão de dois fatores por sugestão de alguns respondentes: poluição do ar e desnível nos bordos das vias (meio-fio).

$\mathrm{Na}$ tabela 2 são elencados os 19 fatores avaliados pelos integrantes da amostra. Para cada um dos fatores elencados havia 5 opçóes (escalas) de resposta no questionário, 
correspondente às opiniōes sobre cada fator: (1) Totalmente sem importância, (2) Pouco importante, (3) Sem opiniāo, (4) Importante e (5) Muito importante.

TABELA 2 Fatores que podem influenciar na escolha da rota pelos ciclistas

\begin{tabular}{|l|l|}
\hline \multicolumn{1}{|c|}{ FATOR } & \multicolumn{1}{c|}{ DEFINIÇÃo } \\
\hline 1. Existência de ciclovias ou ciclofaixas & Infraestrutura cicloviária \\
\hline 2. Pavimento em bom estado de conservação & Qualidade do pavimento \\
\hline 3. Ruas sem aclives (subidas) & Inclinaçóes na via \\
\hline $\begin{array}{l}\text { 4. Presença de estacionamentos de automóveis } \\
\text { no lado direito da rua }\end{array}$ & Estacionamento lateral \\
\hline $\begin{array}{l}\text { 5. Necessidade de atravessar pontes, túneis, viadutos, } \\
\text { rodovias e ferrovias }\end{array}$ & Barreiras urbanas \\
\hline 6. Ruas com apenas uma faixa de tráfego & Largura da via \\
\hline $\begin{array}{l}\text { 7. Muitos cruzamentos com semáforos e/ou sinais de } \\
\text { PARE (a cada } 100 \text { m) }\end{array}$ & Sinalizaçóes de parada frequentes \\
\hline 8. Ruas com velocidade permitida de até 40 km/h & Velocidade de tráfego na via \\
\hline 9. Poucos veículos & Volume de veículos na via \\
\hline 10. Tráfego de ônibus e caminhóes & Via com veículos pesados \\
\hline 11. Iluminação (à noite) & Iluminação pública noturna \\
\hline 12. Arborização (sombra) & Presença de vegetação de grande porte (árvores) \\
\hline 13. Caminho mais rápido & Tempo de viagem \\
\hline 14. Caminho mais curto & Distância de viagem \\
\hline 15. Segurança (criminalidade) & Sensação de segurança/seguridade pessoal \\
\hline 16. Mão única de direção & Vias com apenas um sentido de tráfego \\
\hline 17. Desnível nos bordos (cantos) das ruas (meio-fio) & Desnivelamento dos canais de drenagem \\
\hline 18. Presença de pontos de parada de ônibus & Locais de embarque e desembarque de passageiros \\
\hline 19. Poluição do ar & Emissôes atmosféricas de veículos motorizados \\
\hline
\end{tabular}

FONTE ELABORAÇÃo PRÓPRIA

\section{Aplicação do questionário}

A aplicaçáo dos questionários ocorreu de julho a dezembro de 2016, com ciclistas em viagens utilitárias encontrados em 13 PGV's - estabelecimentos comerciais, como supermercados, lojas esportivas, locais de venda de material de construção, e demais comércios, além de prestadores de serviços, como restaurantes, e instituiçóes de ensino - das regióes Oeste, Centro e Sul da cidade de São José dos Campos, município brasileiro com uma taxa de urbanização de 97,6\% (Prefeitura Municipal de São José do Campos [PMSJc], 2012), situado a cerca de $90 \mathrm{~km}$ da capital do estado, São Paulo (ibGe, 2016).

Entre 2000 e 2010 houve um aumento de $78 \%$ na frota veicular da cidade. No mesmo período, a taxa de motorização passou de 2,91 hab./veículo para 1,91 hab./veículo, praticamente um veículo para cada dois habitantes (IPPLAN, 2014). A síntese da caracterização da cidade é expressa a seguir, na tabela 3. 
TABela 3 | Caracterização de São José dos Campos-sP

\begin{tabular}{|l|c|}
\hline População (habitantes) & 695.992 \\
\hline Área $\left(\mathrm{km}^{2}\right)$ & $1.099,41$ \\
\hline Densidade demográfica $\left(\right.$ hab./ $\left./ \mathrm{km}^{2}\right)$ & 572,96 \\
\hline Extensão do sistema viário $(\mathrm{km})$ & 2.900 \\
\hline Taxa de motorização $($ hab./veículos) & 1,91 \\
\hline Infraestrutura cicloviária $(\mathrm{km})$ & 84,5 \\
\hline
\end{tabular}

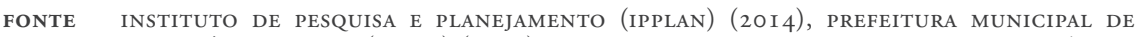
SÃo JOSÉ DO CAMPOS (PMSJC) (20I5), INSTITUTO BRASILEIRO DE GEOGRAFIA E ESTATÍSTICA (IBGE) (2OI6)

Da extensão total do sistema viário, cerca de $2.150 \mathrm{~km}$ estão inseridos no perímetro urbano, e 2,9\% correspondem a infraestruturas cicloviárias, majoritariamente compostas por ciclovias $(65,1 \%)$, seguido por ciclofaixas $(23,9 \%)$ e calçadas compartilhadas $(11 \%)$. Além de reduzidas, as infraestruturas cicloviárias existente possuem diversas descontinuidades identificadas, não estando completamente interligadas umas às outras.

A divisão modal no município demonstra a limitada utilização da bicicleta como modo de transporte $(2,58 \%)$, havendo uma predominância dos modos individuais motorizados (46,54\%), seguido pelos modos coletivos $(27,06 \%)$. Pedestres e outros correspondem a $23,38 \%$ e $0,44 \%$ da distribuição, respectivamente (Instituto de Pesquisa e Planejamento [ipplan], 2014).

Conforme dados do Relatório Geral 2014, do Sistema de Informaçóes da Mobilidade Urbana da Associação Nacional de Transportes Públicos (ANTP, 2016), a quantidade de viagens por transporte individual motorizado (46,54\%) está acima do esperado para municípios de mesmo porte $-34,2 \%$ para municípios de 500 a 1.000 mil habitantes.

Em fevereiro de 2016 o projeto do Plano de Mobilidade Urbana - Plannob da cidade, formulado a partir de 2014, foi aprovado pelo Legislativo. Com isso, o município passou a contar com uma Política Municipal de Mobilidade Urbana, baseada na Lei Federal no 12.587/12 (Brasil, 2012).

\section{Análise dos dados}

Após codificação e tabulação dos dados brutos em planilhas do Excel, foram realizadas análises descritivas (distribuição de frequências, medidas de tendência central e de dispersão) e inferenciais (testes não paramétricos) das respostas.

A interpretação dos dados por meio de técnicas de estatística descritiva resultou em tabelas expositivas sobre: (a) distribuição de homens e mulheres na amostra; (b) idade dos participantes, agrupada por faixas etárias; (c) frequência de uso da bicicleta como meio de deslocamento; e (d) tempo médio de uso da bicicleta como modo de transporte.

Essas informaçóes foram comparadas com os dados da pesquisa o/D, realizada em 2011 no município. Adicionalmente, para facilitar a identificação dos aspectos mais 
relevantes ou expressivos, foram elencadas as proporçóes percentuais de resposta em cada uma das categorias referentes aos fatores que influenciam na escolha das rotas.

Mediante técnicas de estatística inferencial (teste de Kruskal-Wallis), buscou-se identificar possíveis diferenças na avaliação da influência dos diversos fatores entre os agrupamentos de respondentes, com a verificação da existência de eventuais diferenças estatisticamente significativas de opinião em função das características dos perfis (faixa etária, frequência e tempo de uso da bicicleta como meio de transporte).

No teste de Kruskal-Wallis, equivalente não paramétrico da ANOva, não são feitas suposições sobre a distribuição da população, não se assumindo, portanto, que a distribuição possa ser completamente descrita pelos parâmetros média e desvio padrão (como ocorre em uma distribuição normal). As hipóteses em teste no Kruskal-Wallis são de que os grupos têm a mesma distribuição de valores (hipótese nula), ou não têm a mesma distribuição de valores (hipótese alternativa).

Para utilização do mencionado teste, os dados são ordenados conjuntamente (em ranking), com atribuição de posições ascendentes. Existindo empates, a média das colocaçôes empatadas é feita e, na sequência, somam-se as posiçôes de cada grupo, realizando o cálculo de uma fórmula que representa, basicamente, a variância das posiçóes entre os grupos, com um ajuste para o número de empates (McDonald, 2017).

Nesta pesquisa, o mencionado teste estatístico foi realizado no editor de planilhas Excel, por meio da instalação de um suplemento disponível no software gratuito Real Statistics Using Excel (Zaiontz, 2017).

\section{Resultados}

Os resultados descritos neste estudo fazem parte de uma pesquisa mais ampla, sobre opinióes e comportamentos de viagem de ciclistas, que também inclui, além do questionário tratado, o registro e avaliação de rotas percorridas por ciclistas em viagens utilitárias. Portanto, além de responderem ao questionário, os integrantes deste estudo se dispuseram a gravar voluntariamente, durante uma semana, seus percursos em aparelhos GPS. Com isso, obteve-se uma amostra de apenas 32 ciclistas, visto a dificuldade em encontrar participantes dispostos a gravarem seus trajetos, devido à desconfianças iniciais quanto à gravação dos percursos, além da burocrática obtenção de autorizaçóes para aplicação da pesquisa com menores de idade em instituições de ensino.

Na tabela 4 constam as características gerais dos 32 ciclistas que participaram da pesquisa.

TABELA 4 Características dos ciclistas

\begin{tabular}{|l|c|l|l|l|r|}
\hline \multicolumn{2}{|c|}{ Ĝ̂NERO } & \multicolumn{2}{c|}{ FAIXA ETÁRIA } & \multicolumn{2}{c|}{ FREQUÊNCIA DE USO } \\
\hline Masculino & $31(97 \%)$ & $<18$ anos & $7(22 \%)$ & 1 dia por semana & $0(0 \%)$ \\
\hline Feminino & $1(3 \%)$ & 18 a 25 anos & $8(25 \%)$ & 2 a 3 dias por semana & $3(9 \%)$ \\
\hline & & 26 a 35 anos & $13(41 \%)$ & 4 ou mais dias por semana & $29(91 \%)$ \\
\hline & & 36 a 45 anos & $2(6 \%)$ & & \\
\hline & & $>45$ anos & $2(6 \%)$ & & \\
\hline
\end{tabular}

(continua) 
(continuaçâo)

\begin{tabular}{|l|r|l|l|}
\hline \multicolumn{2}{|c|}{ TEMPO DE USO } & \multicolumn{2}{c|}{ MOTIVO } \\
\hline$<3$ meses & $3(9 \%)$ & Trabalho & $19(59 \%)$ \\
\hline 3 a 6 meses & $2(6 \%)$ & Estudo & $13(41 \%)$ \\
\hline 6 meses a 2 anos & $12(38 \%)$ & & \\
\hline 2 a 5 anos & $5(16 \%)$ & & \\
\hline$>5$ anos & $10(31 \%)$ & & \\
\hline
\end{tabular}

FONTE ELABORAÇÃo PRÓPRIA

Há uma porcentagem maior de viagens por motivo 'trabalho', além da predominância de jovens adultos (com até 35 anos) do gênero masculino na amostra, que utilizam a bicicleta mais de 3 dias por semana, em sua maioria, com a prevalência de uso sendo superior a 6 meses.

Comparando as características dos respondentes do questionário com o perfil dos ciclistas identificados na pesquisa o/D verifica-se:

a. Predomínio de ciclistas homens, tanto na pesquisa o/D (76\%) quanto na amostra deste estudo (97\%);

b. Embora a estratificação das faixas etárias seja diferente entre as duas pesquisas, a maioria dos ciclistas é jovem, com até 35 anos - $88 \%$ nesta pesquisa e $61 \%$ na pesquisa $\mathrm{O} / \mathrm{D}$;

c. Foco desta pesquisa, as viagens utilitárias foram, em sua maioria, por motivo de trabalho (59\%). Na amostra da pesquisa o/D, aproximadamente $40 \%$ das viagens por bicicleta tiveram como motivo trabalho ou estudo, e cerca de $50 \%$ tiveram a residência como motivo. De tal forma que cerca de $90 \%$ das viagens na pesquisa $\mathrm{O} / \mathrm{D}$ foram utilitárias (o motivo 'residência' correspondeu à volta para casa após o expediente).

Depreende-se um proeminente viés utilitário das viagens realizadas por ciclistas na cidade de São José dos Campos-sp. Fato apontado em ambas as pesquisas, assim como um perfil de usuários com características similares, embora a aplicação dos questionários tenha ocorrido em PGVs, com amostra do tipo 'bola de neve', e a pesquisa o/D no âmbito domiciliar.

\section{Importância dos fatores para a escolha da rota}

A quantidade e a porcentagem de respostas sobre a importância atribuída pelos ciclistas a cada um dos fatores sáo mostradas na tabela 5, com agrupamento dos níveis de importância da seguinte forma: 'Importante' (incluindo os níveis 'importante' e 'muito importante'), 'Indiferente' (nível 'sem opiniāo') e 'Sem importância' (incluindo os níveis: 'totalmente sem importância' e 'pouco importante’). 
TABELA 5 | Importância dos fatores para escolha da rota

\begin{tabular}{|l|c|c|c|}
\hline \multicolumn{1}{|c|}{ FATORES } & SEM IMPORTÂNCIA & INDIFERENTE & IMPORTANTE \\
\hline Estacionamento & $14(43,8 \%)$ & $9(28,1 \%)$ & $9(28,1 \%)$ \\
\hline Parada de ônibus & $13(40,6 \%)$ & $9(28,1 \%)$ & $10(31,3 \%)$ \\
\hline Velocidade < $40 \mathrm{~km} / \mathrm{h}$ & $13(40,6 \%)$ & $4(12,5 \%)$ & $15(46,9 \%)$ \\
\hline Ruas sem aclives (subidas) & $13(40,6 \%)$ & $2(6,3 \%)$ & $17(53,1 \%)$ \\
\hline Mão única de direção & $13(40,6 \%)$ & $2(6,3 \%)$ & $17(53,1 \%)$ \\
\hline Cruzamentos & $12(37,5 \%)$ & $2(6,3 \%)$ & $18(56,3 \%)$ \\
\hline Pontes, túneis, etc. & $10(31,3 \%)$ & $7(21,9 \%)$ & $15(46,9 \%)$ \\
\hline Ônibus e caminhóes & $11(34,4 \%)$ & $4(12,5 \%)$ & $17(53,1 \%)$ \\
\hline Apenas uma faixa & $7(21,9 \%)$ & $5(15,6 \%)$ & $20(62,5 \%)$ \\
\hline Desnível no meio fio & $6(18,8 \%)$ & $5(15,6 \%)$ & $21(65,6 \%)$ \\
\hline Poluição & $6(18,8 \%)$ & $9(28,1 \%)$ & $17(53,1 \%)$ \\
\hline Ciclovias / ciclofaixas & $8(25 \%)$ & $2(6,3 \%)$ & $22(68,8 \%)$ \\
\hline Poucos veículos & $9(28,1 \%)$ & $0(0 \%)$ & $23(71,9 \%)$ \\
\hline Arborização & $8(25 \%)$ & $0(0 \%)$ & $24(75 \%)$ \\
\hline Pavimento em bom estado & $4(12,5 \%)$ & $1(3,1 \%)$ & $27(84,4 \%)$ \\
\hline Caminho mais curto & $5(15,6 \%)$ & $0(0 \%)$ & $27(84,4 \%)$ \\
\hline Segurança pessoal & $3(9,4 \%)$ & $4(12,5 \%)$ & $25(78,1 \%)$ \\
\hline Iluminação & $3(9,4 \%)$ & $3(9,4 \%)$ & $26(81,3 \%)$ \\
\hline Caminho mais rápido & $4(12,5 \%)$ & $0(0 \%)$ & $28(87,5 \%)$ \\
\hline
\end{tabular}

FONTE ELABORAÇÃo PRÓPRIA

Para facilitar a visualização e interpretação dos resultados, na figura 1 os dados indicados na tabela 5 são apresentados graficamente.

Pela interpretação da figura 1 verifica-se que, dentre os fatores de maior importância, estão características da viagem (caminho mais rápido e mais curto), características do ambiente (iluminação e segurança pessoal) e características físicas da via (conservação do pavimento).

Nesta pesquisa, o caminho mais rápido foi o fator considerado de maior relevância para a escolha da rota, refletindo resultados de diversos outros estudos com ciclistas em viagens utilitárias, em que o menor tempo de viagem frequentemente aparece como de grande importância (Stinson \& Bhat, 2003; Sener, Eluru \& Bhat, 2009; Yang \& Mesbah, 2013; Hrncir et al., 2014).

Como os ciclistas em viagens utilitárias têm horários a cumprir, assume-se que o tempo de viagem é de grande relevância para eles. Sendo assim, se for dada a chance de escolha entre duas rotas com tempos de percurso diferentes, os ciclistas em viagens utilitárias optaráo pela rota mais rápida, a menos que a rota mais longa ofereça atrativos melhores como, por exemplo, maior sombreamento, menor quantidade de subidas, menores volumes e velocidades de tráfego, etc. Vasta bibliografia registra o tempo adicional que os ciclistas estariam dispostos a despender para percorrer determinadas instalaçóes cicloviárias ou rotas com características diversas 
(Abraham, McMillan, Rownlee \& Hunt, 2002; Broach, Gliebe Dill, 2009, 2012; Caulfield, Brick \& NcCarthy, 2012; Krenn, Oja \& Titze, 2014; Krizek, 2006; Krizek, El-Geneidy \& Thompson, 2007; Larsen \& El-Geneidy, 2011; Stinson \& Bhat, 2003, 2004; Tilahun, Levinson \& Krizek, 2007; Winters, Teschke, Grant, Setton \& Brauer, 2010).

\section{FIGURA I Importância dos fatores para escolha da rota}

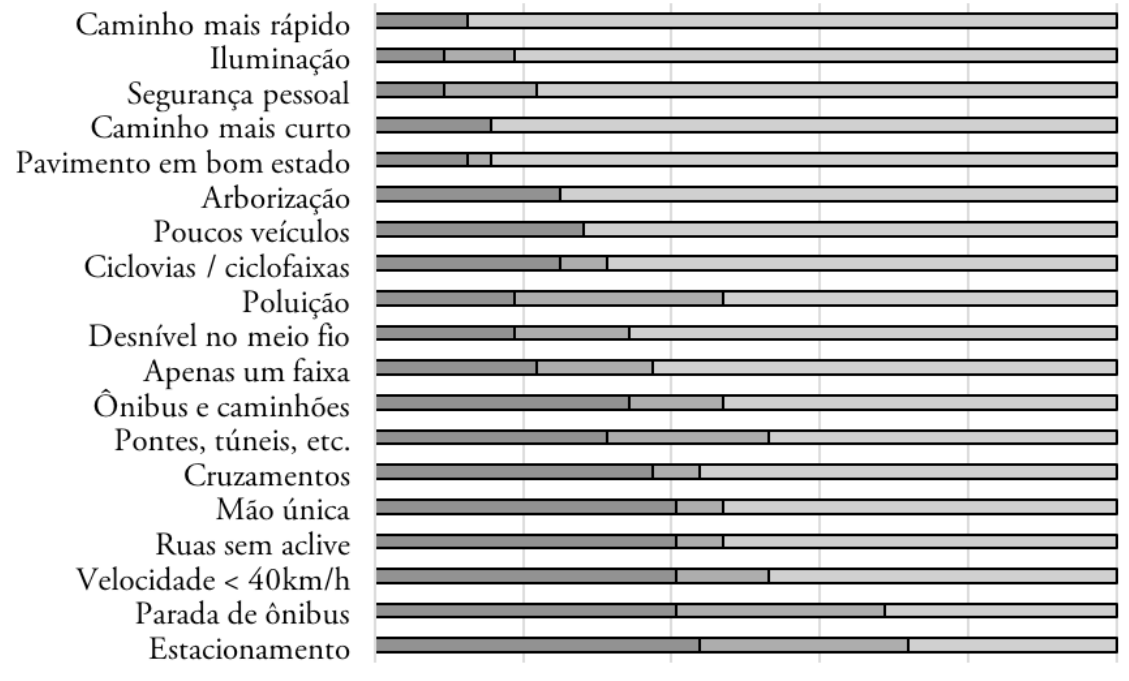

口Sem importância $\quad \square$ Indiferente $\quad$ Importante

FUENTE ELABORAÇÃO PRÓPRIA

É pertinente destacar que, embora sejam tratados de modo similar, a distância e o tempo de viagem não são diretamente proporcionais, dado que o tempo de viagem não é constante - os ciclistas náo viajam a uma velocidade fixa durante todo o trajeto (Beheshtitabar et al., 2014), devido à necessidade de maior esforço físico (Zhao, 2014) em decorrência de alteraçóes na declividade de determinados trechos do percurso, além da influência de características da rota, como tempo de espera em interseçôes sinalizadas (Ehrgott, Wang, Raith \& Van Houtte, 2012), e de características do usuário, como a experiência (El-Geneidy, Krizek \& Iacono, 2007).

Por isso houve a separação dos fatores tempo e distância no questionário (representados nesta pesquisa pelos fatores "caminho mais rápido" e "caminho mais curto", respectivamente), e certo distanciamento dos mesmos nos resultados mostrados na figura 1.

Deduz-se que os ciclistas da amostra preferem trajetos curtos, conforme também apontado pelos estudos de Abraham et al. (2002), Broach et al. (2011), Dill (2009) e Hood, Sall y Charlton. (2011), mas priorizam os caminhos mais rápidos, o que pode acarretar na ocasional escolha de percursos mais longos e atrativos. Nesse sentido, Winters et al. (2010), por exemplo, constataram a ocorrência de viagens 
cicloviárias por caminhos mais longos, com significativamente maior quantidade de cobertura vegetal (arborização) dos que os menores caminhos possíveis.

Considerada importante por $75 \%$ dos respondentes do questionário, a arborização está intrinsecamente relacionada à qualidade do ar (Nicodemo \& Primavesi, 2009), fator representado nesta pesquisa como "poluição do ar", e considerado importante por 53\% dos ciclistas da amostra. Dentre os estudos encontrados referentes à poluição, destaca-se uma pesquisa realizada por Hatzopoulou et al. (2013) em Montreal-Canadá, com uso de uma ferramenta para medição da exposição a determinados poluentes de tráfego. No mencionado estudo, $57 \%$ das rotas percorridas pelos ciclistas possuíam um trajeto alternativo com menores índices de exposição aos poluentes, mas apenas em $43 \%$ das viagens os menores caminhos eram, também, os com menores índices de exposição aos poluentes.

Não foram encontrados estudos que se referissem, especificamente, à influência da poluição do ar na escolha da rota pelos ciclistas, apenas pesquisas sobre a atuação deste atributo na opção pelo modo de transporte cicloviário. Além de um estudo sobre os benefícios da atividade física desenvolvida durante o transporte ativo (caminhar e pedalar para se deslocar), que superam os malefícios da exposição a níveis elevados de poluentes do ar em grande parte das cidades no mundo, mesmo com muitas horas de exposição (Alisson, 2016; Cepeda et al., 2016).

O segundo e o terceiro fatores elencados na figura 1 estão relacionados pois, além da percepção de segurança (risco de assaltos e agressôes) associar-se à presença de iluminação no período noturno (Sener et al., 2009), a iluminação pública também funciona como "medida preventiva importante à diminuição da criminalidade", sendo "responsável pela diminuição significativa de assaltos e latrocínios" (Brasil, 2007, p. 154). A segurança também apareceu próxima do quesito iluminação na média geral de importância dos fatores na pesquisa de Segadilha e Sanches (2014b).

Em um estudo sobre atributos para o projeto de redes cicloviárias, estes dois fatores foram considerados importantes por ciclistas e especialistas (Pitilin, 2016). A influência e relevância da segurança pessoal na escolha da rota aponta para a "percepção de insegurança que existe atualmente nas cidades brasileiras", segundo Pitilin e Sanches (2016, p. 11).

Sobre o quinto fator (conservação do pavimento), foram corroborados resultados de estudos anteriores, em que ciclistas evitaram circular por vias sem pavimentação ou com pavimento degradado (Segadilha \& Sanches, 2014b, 2014a; Winters \& Teschke, 2010), e preferiram superfícies lisas (Antonakos, 1994; Bernhoft \& Carstensen, 2008; Stinson \& Bhat, 2003, 2004). Algo igualmente embasado pela pesquisa de Pitilin (2016), em que a conservação do pavimento foi considerada muito importante para o projeto de uma rede cicloviária por ciclistas e especialistas na área.

Adicionalmente, Aultman-Hall, Hall e Baetz (1997) notaram que os ciclistas em viagens utilitárias frequentemente percorrem caminhos bem qualificados (amplos, com superfície de boa qualidade, largos e acessíveis). E Kang e Fricker (2013) constataram que a aversão a superfícies de menor qualidade supera o risco de conflitos com o fluxo de pedestres, podendo levar ciclistas a percorrer passeios públicos (calçadas) para evitar o compartilhamento com veículos em vias com pavimentação de baixa qualidade. 
Salientam-se dois outros fatores comumente considerados como de grande relevância na escolha da rota em diversos outros estudos: velocidade permitida na via e declividade (representados por "velocidade $<40 \mathrm{~km} / \mathrm{h}$ " e "ruas sem aclives subidas" nesta pesquisa). Aproximadamente $40 \%$ dos respondentes consideraram sem importância os fatores velocidade do tráfego e declividade. Ainda assim, 47\% e 53\% dos participantes consideraram a velocidade e a declividade importantes, respectivamente.

Na literatura consultada há um indicativo de preferência pela circulação em vias com limites de velocidade reduzidos (Sener et al., 2009), evitando vias principais onde os veículos trafegam em alta velocidade (Krenn et al., 2014). Caulfield et al. (2012), por exemplo, em uma pesquisa aplicada em Dublin (Irlanda), demonstraram a preferência dos ciclistas por vias com velocidades reduzidas (30 ao invés de 50 ou $80 \mathrm{~km} / \mathrm{h}$ ).

A declividade também aparece, frequentemente, dentre os fatores mais comumente associados à escolha da rota, e é vista como um componente desfavorável quando os ciclistas qualificam uma rota preferencial, sendo constatada uma tendência de evitar percursos íngremes (Hood et al., 2011; Menghini, Carrasco, Schüssler \& Axhausen., 2010), mesmo que para isso tenham que percorrer um caminho mais longo (Krenn et al., 2014). Complementarmente, Li, Wang, Liu e Raglan (2012) identificaram uma associação negativa do fator "inclinação" com a sensação de conforto ao pedalar, resultado atribuído ao maior esforço físico (energia necessária para superar as subidas).

Assim como o fator poluiçáo do ar, o desnível nos bordos das vias (meio-fio) foi adicionado ao questionário por sugestão de respondentes da pesquisa-piloto, sendo digno de nota pois, apesar de não terem sido encontrados estudos que relacionassem este fator à escolha de rota, aproximadamente $66 \%$ dos respondentes o consideraram importante. É possível relacioná-lo com a qualidade do pavimento, visto o constatado desnivelamento dos canais de drenagem nas cidades brasileiras (atribuído às práticas de recapeamento asfáltico vigentes). De modo que é comum que "os bordos das vias, na maioria das cidades brasileiras, apresentem sarjetas mal construídas ou em estado de deterioração, com fissuras alastrando-se para além do bordo do pavimento" (Brasil, 2007, p. 125).

No que diz respeito à infraestrutura cicloviária, sua presença é de grande relevância para a escolha da rota entre ciclistas em viagens utilitárias (Raford, Chiaradia \& Gil, 2007), sendo apontada como possível contribuição significativa para experiências positivas ao pedalar (Snizek, Nielsen \& Skov-Petersen, 2013). A existência de infraestrutura cicloviária pode até ser capaz de diminuir o custo (visto como oneroso) de percorrer vias arteriais, conforme estudo desenvolvido por Abraham et al. (2002), em Calgary (Canadá). No presente estudo, 69\% dos ciclistas consideraram a presença de ciclovias/ciclofaixas como relevante na escolha de rota, algo também verificado por Broach et al. (2009). Ademais, a existência destas estruturas foi apontada como muito importante por ciclistas e especialista que participaram da pesquisa de Pitilin (2016).

Notabiliza-se a pouca importância atribuída aos fatores referentes aos pontos de parada de ônibus e estacionamentos de automóveis. Ainda que indiretamente, a 
presença de pontos de parada de ônibus relaciona-se à conservação do pavimento, pois a incorporação de baias com pavimento rígido (mais resistente) é exceção no país. Logo, a opção pelo pavimento asfáltico ocasiona frequentes deformaçóes nos locais de parada de ônibus para embarque e desembarque de passageiros. No que concerne aos estacionamentos, existem duas tipologias usuais (paralelo ou em ângulo). Em ambos os tipos o risco de conflitos com ciclistas é razoável, pela obstrução da visibilidade na saída da vaga, ou durante a abertura de portas dos veículos.

Comparação da importância atribuída aos fatores por diferentes estratos da amostra Não obstante houvesse, a princípio, a intenção de se fazer uma análise sobre a existência de eventuais diferenças estatisticamente significativas de opinião em relação ao gênero, a ínfima quantidade de ciclistas mulheres na amostra impossibilitou tal procedimento. Essa reduzida incidência feminina no transporte cicloviário foi auferida por diversos autores, que constataram o predomínio de ciclistas masculinos nas pesquisas que desenvolveram (Garrard, 2003; Krizek, Johnson \& Tilahun, 2004; Prati, 2017). Este fato é indicativo da baixa representatividade do ponto de vista feminino na concepção de políticas públicas 'cicloinclusivas', com omissão do indispensável papel das mulheres como indicadores de qualidade urbana, ao usarem a bicicleta como meio de transporte cotidiano somente em condiçóes consideradas ideais - algo reforçado pela equidade de gênero na distribuição de viagens cicloviárias em países europeus com elevadas taxas de uso da bicicleta e amplas redes cicloviárias (Garrard, Rose \& Lo, 2008).

\section{a) Com relação à faixa etária}

A idade pode interferir na escolha da rota, ainda que de modo indireto, em funçáo das habilidades físicas que se transformam com o envelhecimento, bem como em virtude do tempo de reação/agilidade dos usuários, que se altera ao longo dos anos (Bernhoft \& Carstensen, 2008).

Diferenças de opinião entre as faixas etárias foram avaliadas por intermédio do teste de Kruskal Wallis. Quando os valores de p (probabilidade) obtidos por este teste são inferiores a 0,05 , constata-se a existência de diferenças estatisticamente significativas de opinião. Para apenas 4 (conservação do pavimento, iluminação, comprimento da viagem, e segurança), dos 19 fatores analisados, essa diferença foi constatada $(\mathrm{p}<0,05)$.

Não se pode afirmar quais seriam as diferenças de opinião entre os grupos etários, apenas indicar a existência das mesmas, impossibilitando a confirmação dos apontamentos de Bernhoft e Carstensen (2008), por exemplo, que conjecturaram que a preferência dos ciclistas mais jovens por superfícies lisas deveu-se ao maior apreço pelo ganho de velocidade, visto que uma superfície irregular é um impedimento para adquirir maiores velocidades (preferenciais entre jovens).

\section{b) Com relaçáo ao tempo de uso da bicicleta}

A experiência pode influenciar diretamente na preferência por rotas. Para Stinson e Bhat (2004), por exemplo, a regularidade do pavimento possui um menor efeito sobre a escolha da rota entre indivíduos inexperientes, enquanto que ciclistas experientes possuem maior sensibilidade ao tempo de viagem. 
Por meio do teste de Kruskal Wallis verificou-se que 5 fatores (conservação do pavimento, iluminação, arborização, duração da viagem, e segurança), dos 19 analisados, apresentaram diferenças estatisticamente significativa de opinião em função do tempo de uso da bicicleta $(\mathrm{p}<0,05)$.

Pitilin (2016) também constatou diferenças de opinióes entre ciclistas com diferentes níveis de experiência (tempo de uso da bicicleta) em relação aos fatores arborização e segurança. Cabe ressalva que a mencionada autora utilizou um teste paramétrico (análise de variância - ANOvA) para testar hipóteses sobre a igualdade da média de duas ou mais populaçóes.

Reitera-se que, embora seja possível apontar a existência de diferenças estatisticamente significativas em relação à conservação do pavimento nesta pesquisa, os resultados da análise não permitem corroborar os apontamentos de Stinson e Bhat (2004), por exemplo. Sendo inviável, portanto, afirmar quais seriam estas diferenças de opinião entre os grupos, apenas indicar a existência das mesmas entre ciclistas experientes e inexperientes.

Por fim, salienta-se que em ambas as classificaçôes de perfis (faixa etária e tempo de uso da bicicleta), o fator "poucos veículos", referente ao volume do tráfego, obteve um p (probabilidade) de 0,06, bem próximo, mas, ainda assim, superior ao limiar para afirmação de existência de diferenças entre os grupos $(\mathrm{p}<0,05)$. Tal fator é negativamente associado à percepção de conforto dos ciclistas, em função do risco de colisóes e acidentes, além dos congestionamentos ocasionados em função do fluxo intenso de veículos ( $\mathrm{Li}$ et al., 2012).

\section{Conclusão}

O reconhecimento dos fatores que influenciam na escolha das rotas, com base na opinião dos usuários da bicicleta, possibilita determinar os aspectos prioritários a serem considerados no planejamento de uma rede cicloviária. Neste estudo, evidenciaram-se justamente os principais fatores que influenciam na escolha de rota de ciclistas em viagens utilitárias. As informaçóes apresentadas compreendem a opiniáo de uma pequena amostra de ciclistas voluntários, que realizam viagens utilitárias regularmente em uma cidade brasileira de porte médio.

Por intermédio da análise das respostas dos questionários, tornou-se claro que a prioridade dos usuários da amostra é chegar o mais rapidamente possível a seus destinos, por caminhos iluminados e seguros, além de pedalar o minimamente necessário, de preferência por vias com pavimento em bom estado de conservação.

Os resultados desta pesquisa são diametralmente opostos da noção usual de que apenas a construção de ciclovias fragmentadas resolve todos os problemas de deslocamento enfrentados pelos ciclistas. Eles direcionam para a disseminação de redes cicloviárias integradas, com rotas coesas e diretas que minimizem os tempos de viagem, o comprimento dos percursos, e os desvios de conexáo entre origens e destinos, promovendo ligação direta entre pontos de interesse. Além de contarem com especificaçóes mínimas para integridade e conforto do usuário (iluminação, segurança pública/viária, conservação adequada do pavimento e arborização). Entretanto, a dificuldade em encontrar tais características nas cidades brasileiras, que as 
restringem, no máximo, às infraestruturas cicloviárias destinadas ao lazer, ilustram o patamar de prioridade em que se encontra o transporte cicloviário no país.

As observaçóes deste estudo não refletem, necessariamente, as preferências de ciclistas potencias ou ocasionais, que possivelmente diferem das opinióes dos ciclistas regulares. Futuras análises mais detalhadas sobre as opinióes de ciclistas com diferentes tempos de uso da bicicleta - utilizado como variável proxy para o nível de experiência como ciclista, podem contribuir para o planejamento de redes cicloviárias mais atrativas para pessoas que ainda não fazem uso da bicicleta como meio de transporte, visto a maior proximidade entre opinióes de ciclistas inexperientes e usuários potenciais.

Quanto a novos estudos sobre os fatores que influenciam na escolha da rota dos ciclistas, baseados na opinião destes usuários, é cabível mencionar a relevância da inclusão de itens tradicionalmente não abordados, mas apontados como importantes pelos próprios ciclistas, bem como a possibilidade de inclusão de outras condicionantes do ambiente, como poluição sonora e sensação térmica. Além do aprofundamento na análise da influência de atributos já abordados, de modo que seria válido realizar, por exemplo, mediçóes in loco dos níveis de poluentes das rotas escolhidas.

Dentre as limitaçóes do estudo, aponta-se para o reduzido número de mulheres na amostra de ciclistas, impossibilitando análises estatísticas inferenciais para verificação de eventuais diferenças significativas de opinião em função do gênero dos usuários. Esse fato evidencia a existência de lacunas neste e em outros estudos sobre viagens cicloviárias utilitárias, especificamente no que concerne à predominante influência masculina nas amostras obtidas, abrindo oportunidade para estudos futuros sobre a participação feminina no transporte cicloviário, bem como suas opiniôes e experiências como ciclistas em viagens utilitárias.

Como defasagem desta pesquisa, salienta-se não ter aparecido o fator "sentido da via” na revisão bibliográfica, apesar de sua inclusão no questionário (mão única de direção). Além disso, dentre os aspectos que poderiam ser aprimorados, tem-se o quesito quantidade de veículos, ao qual não foi atribuído um valor objetivo, tornando abstrata e subjetiva a avaliaçáo do fator "poucos veículos", assim como o item referente à qualidade do pavimento, em que a formulação da questão (pavimento em bom estado de conservação) pode ter induzido o respondente, possivelmente direcionando as respostas e interferindo nos resultados.

Por fim, cabe frisar a inserção implícita do fator 'segurança viária' nos itens relacionados à quantidade de veículos ('poucos veículos'), composição e velocidade do tráfego ('tráfego de ônibus e caminhôes' e 'ruas com velocidade permitida de até $40 \mathrm{~km} / \mathrm{h}$ ', respectivamente), possibilitando aferir sua importância de modo indireto. 


\section{Referências bibliograficas}

Abraham, J. E., McMillan, S., Rownlee, A. T. \& Hunt, J. D. (2002, January). Investigation of cycling sensitives. Transportation Research Board Annual Conference, Washington, D.C..

Alisson, E. (2016). Estudo estima impactos do planejamento urbano na saúde. Agência FAPESP. Em http://agencia.fapesp.br/estudo_estima_impactos_do_planejamento_urbano_ na_saude/24124/

Anderson, L. B., Schnohr, P., Schroll, M. \& Hein, H., O. (2000). All-cause mortality associated with physical activity during leisure time, work, sports, and cycling to work. Archives of Internal Medicine, 160(11), 1621-1628. https://doi.org/10.1001/ archinte.160.11.1621

Antonakos, C. L. (1994). Environmental and travel preferences of cyclists. Transportation Research Board, (1438), 25-33.

Appolinário, F. (2012). Metodologia da ciência: filosofia e prática da pesquisa. São Paulo: Cengage Learning.

Associação Nacional de Transportes Públicos - ANTP. (2016, julho). Sistema de Informaçôes da Mobilidade Urbana - Relatório Geral 2014. Em http://files.antp.org.br/2016/9/3/ sistemasinformacao-mobilidade--geral_2014.pdf

Aultman-Hall, L., Hall, F. L. \& Baetz, B. B. (1997). Analysis of bicycle commuter routes using Geographic Information Systems: Implications for bicycle planning. Transportation Research Record, 1578(1578), 102-110. https://doi.org/10.3141/1578-13

Baptista, J. R., Monteiro, M. M., Verniers, C. R., Gomes, T. von Z., Santos, R. E., Kyono, L. F. \& Costa, M. da Silva. (2009, setembro/outubro). Metodologia e novos instrumentos para a realizaçáo de pesquisas de origem e destino domiciliares. $17^{\circ}$ Congresso Brasileiro de Transporte e Trânsito, Curitiba, PR, Brasil.

Bassett, D. R., Pucher, J., Buehler, R., Thompson, D. L. \& Crouter, S. E. (2008). Walking, cycling, and obesity rates in Europe, North America, and Australia. Journal of Physical Activity and Health, 5(6), 795-814. Em http://www.cycle-helmets.com/walk-bikeobesity-rates.pdf

Beheshtitabar, E., Ríos, S. A., König-Hollerwöger, D., Svatý, Z.; \& Rydergren, C. (2014). Route choice modelling for bicycle trips. International Journal for Traffic and Transport Engineering, 4(2), 194-209. https://doi.org/10.7708/ijtte.2014.4(2).06

Bernhoft, I. M. \& Carstensen, G. (2008). Preferences and behavior of pedestrians and cyclists by age and gender. Transportation Research Part F, 11(2), 83-95.

Brasil. (2007). Programa Brasileiro de Mobilidade por Bicicleta - Bicicleta Brasil. Caderno de referência para elaboração de Plano de Mobilidade por Bicicleta nas Cidades. Brasília: Secretaria Nacional de Transporte e da Mobilidade Urbana.

Brasil. (2012). Lei no 12.587, de 3 de janeiro de 2012. Institui as diretrizes da Política Nacional de Mobilidade Urbana; revoga dispositivos dos Decretos-Leis nos 3.326, de 3 de junho de 1941, e 5.405, de 13 de abril de 1943, da Consolidação das Leis do Trabalho (CLT), aprovada pelo Decreto-Lei no 5.452, de 1o de maio de 1943, e das Leis nos 5.917, de 10 de setembro de 1973, e 6.261, de 14 de novembro de 1975; e dá outras providências. Diário Oficial da Uniāo, Brasília, DF. 
Broach, J., Dill, J. \& Gliebe, J. (2012). Where do cyclists ride? A route choice model developed with revealed preference GPs data. Transportation Research Part A: Policy and Practice, 46(10) 1730-1740. https://doi.org/10.1016/j.tra.2012.07.005

Broach, J., Gliebe, J. \& Dill, J. (2009, January). Development of a multi-class bicyclist route choice model using revealed preference data. 12th International Conference on Travel Behavior Research, Jaipur, India.

Broach, J., Gliebe, J. \& Dill, J. (2011). Bicycle route choice model developed using revealed preference GPS data. 90th Annual Meeting of the Transportation Research Board, Washington, D.c. Em https://bit.ly/2Inafco

Casello, J. M., Nour, A. \& Rewa, K. (2011, January). An analysis of stated preference and GPS data for bicycle travel forecasting. TRB 90th Annual Meeting, Washington, D.c.

Casello, J. M., Rewa, K. C., Nour, A. (2012, January). An analysis of empirical evidence of cyclists' route choice and their implications for planning. Transportation Research Board 91st Annual Meeting, Washington, D.c.

Caulfield, B., Brick, E. \& McCarthy, O. T. (2012). Determining bicycle infrastructure preferences - A case study of Dublin. Transportation Research Part D, 17(5), 413-417. https://doi.org/10.1016/j.trd.2012.04.001

Cepeda, M., Schoufour, J., Freak-Poli, R., Koolhaas, C. M., Dhana, K., Bramer, W. M. \& Franco, O. H. (2017). Levels of ambient air pollution according to mode of transport: a systematic review. The Lancet Public Health, 2(1), 23-34. http://doi.org/10.1016/ S2468-2667\%2816\%2930021-4

Dill, J. (2009). Bicycling for transportation and health: The role of infrastructure. Journal of Public Health Policy, 30(1), 95-110. https://doi.org/10.1057/jphp.2008.56

Dill, J. \& Gliebe, J. (2008). Understanding and measuring bicycle behavior: A focus on travel time and route choice. Oregon Transportation Research and Education Consortium (OTREC), Portland, or.

Dora, C. \& Phillips, M. (2000). Transport, environment and health. Copenhagen, Denmark: WHO regional publications. Em http://apps.who.int/iris/bitstream/handle/10665/107336/ E72015.pdf;jsessionid=2270625ED5AA2CCAAF7DF6EEB5F88A58? sequence $=1$

Ehrgott, M., Wang, J. Y. T., Raith, A. \& Van Houtte, C. (2012). A bi-objective cyclist route choice model. Transportation Research Part A, 46(4), 652-663. https://doi. org/10.1016/j.tra.2011.11.015

El-Geneidy, A., Krizek, K. \& Iacono, M. (2007, January). Predicting bicycle travel speeds along different facilities using GPS data: a proof of concept model. TRB 2007 Annual Meeting, Washington, D.C.

Emond, C. \& Handy, S. (2012). Factors associated with bicycling to high school: insights from Davis, CA. Journal of Transport Geography, 20(1) 71-79. https://doi.org/10.1016/j. jtrangeo.2011.07.008

Fajans, J. \& Curry, M. (2001). Why bicyclists hate stop signs. Acess, (18), 21-22. Em http:// www.sfbike.org/download/bike_law/why_bikes_hate_stops.pdf

Garrard, J. (2003). Healthy revolutions: promoting cycling among women. Health Promotion Journal of Australia, 14(3), 213-215.

Garrard, J., Rose, G., Lo, S. K. (2008). Promoting transportation cycling for women: The role of bicycle infrastructure. Preventive Medicine, 46(1), 55-59. https://doi.org/10.1016/j. ypmed.2007.07.010 
Gil, A. C. (2008). Métodos e Técnicas de Pesquisa Social (6a ed.). São Paulo: Atlas.

Godefrooij, T., Pardo, C. \& Sagaris, L. (Eds.). (2009). Cycling-Inclusive Policy Development: A Handbook. Utrecht: Interface for Cycling Expertise (I-CE). Em http://www.fietsberaad. nl/library/repository/bestanden/Cycling-handbook_secure.pdf

Gordon-Larsen, P., Boone-Heinonen, J., Sidney, S., Sternfeld, B., Jacobs, D. R. Jr. \& Lewis, C. E. (2009). Active commuting and cardiovascular disease risk: the CARDIA study. Archives of Internal Medicine, 169(13) 1216-1223. https://doi.org/10.1001/ archinternmed.2009.163

Hamer, M. \& Chida, Y. (2008). Active commuting and cardiovascular risk: A meta-analytic review. Preventive Medicine 46(1) 9-13. https://doi.org/10.1016/j.ypmed.2007.03.006

Hatzopoulou, M., Weichenthal, S., Barreau, G., Goldberg, M., Farrell, W., Crouse, D. \& Ross, N. (2013). A web-based route planning tool to reduce cyclists' exposures to traffic pollution: A case study in Montreal, Canada. Environmental Research, 123, 58-61. https://doi.org/10.1016/j.envres.2013.03.004

Hood, J., Sall, E. \& Charlton, B. (2011). A grs-based bicycle route choice model for San Francisco, California. Transportation Letters: The International Journal of Transportation Research, 3, 63-75. https://doi.org/10.3328/TL.2011.03.01.63-75

Hrncir, J., Song, Q., Zilecky, P. \& Jakob, M. (2014, August). Bicycle route planning with route choice preferences. ECAI'14 Proceedings of the Twenty-first European Conference on Artificial Intelligence, Prague, Czech Republic. https://doi.org/10.3233/978-1-61499419-0-1149

Hunt, J. D. \& Abraham, J. E. (2007). Influences on bicycle use. Transportation, 34(4), 453470. https://doi.org/10.1007/s11116-006-9109-1

Instituto Brasileiro de Geografia e Estatística (IBGE). (2016). Cidades. São Paulo. São José dos Campos. Em http://cidades.ibge.gov.br/xtras/perfil.php?lang=\&codmun=354990\&se arch=sao-paulo|sao-jose-dos-campos

Instituto de Pesquisa, Administração e Planejamento (IPplan). (2014). Atlas da pesquisa origem e destino - panorama da mobilidade em São José dos Campos. São Carlos: Editora Cubo.

Instituto de Políticas de Transporte e Desenvolvimento (ITDP), Brasil. (2017). Guia de Planejamento Cicloinclusivo. Em http://2rps5v3y8o843iokettbxnya.wpengine. netdna-cdn.com/wp-content/uploads/2017/09/guia-cicloinclusivo-ITDP-Brasilsetembro-2017.pdf

Instituto de Políticas para el Transporte y el Desarrollo (ITDP), México. (2011). Manual integral de movilidad ciclista para ciudades mexicanas. Tomo III. Red de Movilidade em Bicicleta. Ciclociudades. Em http://ciclociudades.mx/manual/

Kang, L. \& Fricker, J. D. (2013). Bicyclist commuters' choice of on-street versus off-street route segments. Transportation, 40(5), 887-902. https://doi.org/10.1007/s11116013-9453-x

Koh, P. P. \& Wong, Y. D. (2013). Influence of infrastructural compatibility factors on walking and cycling route choices. Journal of Environmental Psychology, 36, 202-213. https:// doi.org/10.1016/j.jenvp.2013.08.001

Krenn, P., Oja, P. \& Titze, S. (2014). Route choices of transport bicyclists: a comparison of actually used and shortest routes. International Journal of Behavioral Nutrition and Physical Activity, 11(1), 31. https://doi.org/10.1186/1479-5868-11-31 
Krizek, K. J. (2006). Two approaches to valuing some of bicycle facilities' presumed benefits. Journal of the American Planning Assotiation, 72(3), 309-320. https://doi. org/10.1080/01944360608976753

Krizek, K. J., El-Geneidy, A. \& Thompson, K. (2007). A detailed analysis of how an urban trail system affects the travel of cyclists. Transportation, 34(5), 611-624. https://doi. org/10.1007/s11116-007-9130-z

Krizek, K. J., Johnson, P. J. \& Tilahun, N. (2004). Gender differences in bicycling behavior and facility preferences. Conference on Research on Women's Issues in Transportation, Chicago, Illinois.

Land Transport Safety Authority (LTSA). (2004). Cycle network and route planning guide. Em https://www.nzta.govt.nz/assets/resources/cycle-network-and-route-planning/docs/ cycle-network-and-route-planning.pdf

Larsen, J. \& El-Geneidy, A. (2011). A travel behavior analysis of urban cycling facilities in Montréal Canada. Transportation Research Part D, 16(2), 172-177. https://doi. org/10.1016/j.trd.2010.07.011

Li, Z., Wang, W., Liu, P. \& Ragland, D. R. (2012). Physical environments influencing bicyclists' perception of comfort on separated and on-street bicycle facilities. Transportation Research Part D, 17(3), 256-261. https://doi.org/10.1016/j.trd.2011.12.001

McDonald, J. H. (2017). Kruskal-Wallis test. Handbook of Biological Statistics. Em http://www. biostathandbook.com/kruskalwallis.html

Menghini, G., Carrasco, N., Schüssler, N. \& Axhausen, K. W. (2010). Route choice of cyclists in Zurich. Transportation Research Part A, 44(9), 754-765. https://doi.org/10.1016/j. tra.2010.07.008

Milakis, D., Athanasopoulos, K., Vafeiadis, E., Vasileiadis, K. \& Vlastos, T. (2012). Planning of the Athens metropolitan cycle network using participative multicriteria GIS analysis. Procedia - Social and Behavioral Sciences, 48, 816-826. https://doi.org/10.1016/j. sbspro.2012.06.1059

Moudon, A. V., Lee, C., Cheadle, A. D., Collier, C. W., Johnson, D., Schmid, T. L. \& Weather, R. D. (2005). Cycling and the built environment, a us perspective. Transportation Research Part D, 10(3), 245-261. https://doi.org/10.1016/j.trd.2005.04.001

Nicodemo, M. L. F., Primavesi, O. (2009). Por que manter árvores na área urbana?. São Carlos: Embrapa Pecuária Sudeste. Em https://ainfo.cnptia.embrapa.br/digital/bitstream/ CPPSE-2009/18356/1/Documentos89.pdf

Oja, P., Titze, S., Bauman, A., de Geus, B., Krenn, P., Reger-Nash, B. \& Kohlberger, T. (2011). Health benefits of cycling: a systematic review. Scandinavian Journal of Medicin \& Science in Sports, 21(4), 96-509. https://doi.org/10.1111/j.1600-0838.2011.01299.x

Pitilin, T. R. (2016). Identificação dos principais atributos para o projeto de uma rede cicloviária. Dissertação de mestrado, Universidade Federal de São Carlos, São Carlos, sp, Brasil.

Pitilin, T. R. \& Sanches, S. P. (2016, novembro). Identificação dos principais atributos para o projeto de uma rede cicloviária. Anais do XXX ANPET Congresso de Pesquisa e Ensino em Transporte. Rio de Janeiro, RJ, Brasil. Em http://146.164.5.73:20080/ssat/interface/ content/anais_2015/TrabalhosFormatados/RT558.pdf

Prati, G. (2017). Gender equality and women's participation in transport cycling. Journal of Transport Geography. https://doi.org/10.1016/j.jtrangeo.2017.11.003 
Prefeitura Municipal de São José dos Campos (pMsjc). (2012). São José em dados. Informaçōes sobre a cidade de São José dos Campos. Secretaria de Planejamento Urbano. Departamento de Planejamento Urbano. Divisão de Pesquisa. Em https://www.sjc. sp.gov.br/media/293116/saojoseemdados4_fev.pdf

Prefeitura Municipal de São José dos Campos (PMSjc). (2015). Anexo I-Plano estratégico. Plano de Mobilidade Urbana de São José dos Campos - PlanMob Secretaria de Transportes. Em http://planmob.sjc.sp.gov.br/wp-content/uploads/downloads/2a87e178cc2b89fb 6fcfc63af6bf54f7.pdf

Raford, N., Chiaradia, A., Gil, J. (2007, January). Space syntax: The role of urban form in cyclist route choice in Central London. TRB 2007 Annual Meeting CD-ROM. Washington, D.C.

Raia, Jr., A. A. (2000). Acessibilidade e mobilidade na estimativa de um indice de potencial de viagens utilizando redes neurais artificiais e sistemas de informaçōes geográficas. Tese de doutorado, Universidade de São Paulo, São Carlos, sp, Brasil.

Segadilha, A. B. P. (2014). Identificação dos fatores que influenciam na escolha da rota pelos ciclistas: estudo de caso da cidade de São Carlos. Dissertaçáo de mestrado, Universidade Federal de São Carlos, São Carlos, sp, Brasil.

Segadilha, A. B. P. \& Sanches, S. P. (2014a). Identification of factors that influence cyclists' route choice. Procedia - Social and Behavioral Sciences, 160, 372-380. Em https://bit. ly/2GKDLvl

Segadilha, A. B. P. \& Sanches S. P. (2014b). Fatores que influenciam na escolha das rotas pelos ciclistas. Revista dos Transportes Públicos, 1(36), 43-56. Em https://bit.ly/2Gy5qMY

Segadilha, A. B. P. \& Sanches, S. P. (2014c). Análise de rotas de viagens por bicicleta usando GPS e SIG. Anais do XXVIII Congresso de Ensino e Pesquisa em Transporte - ANPET, Curitiba, PR, Brasil.

Segadilha, A. B. P. \& Sanches, S. P. (2014d). Analysis of bicycle commuter routes using GPSs and GIs. Procedia - Social and Behavioral Sciences, 162, 198-207. https://doi. org/10.1016/j.sbspro.2014.12.200

Sener, I. N., Eluru, N. \& Bhat, C. R. (2009). An analysis of bicycle route choice preferences in Texas, us. Transportation, 36(5), 511-539. https://doi.org/10.1007/s11116-0099201-4

Snizek, B., Nielsen, T. A. S. \& Skov-Petersen, H. (2013). Mapping bicyclists' experiences in Copenhagen. Journal of Transport Geography, 30, 227-233. https://doi.org/10.1016/j. jtrangeo.2013.02.001

Stinson, M. A. \& Bhat, C. R. (2003). An analysis of commuter bicyclist route choice using a stated preference survey. Transportation Research Record: Journal of the Transportation Research Board, 1828, 107-115. Em https://pdfs.semanticscholar.org/646e/08bdd318 0da839d93293a9b8e98ee39a036f.pdf

Stinson, M. A. \& Bhat, C. R. (2004, January). A comparison of the route preferences of experienced and inexperienced bicycle commuters. 84th Annual Meeting of Transportation Research Board, Washington, D.c.

Tilahun, N., Levinson, D. M. \& Krizek, K. J. (2007). Trails, lanes, or traffic: Valuing bicycle facilities with an adaptive stated preference survey. Transportation Research Part A, 41(4), 287-301. https://doi.org/10.1016/j.tra.2006.09.007 
Winters, M. \& Teschke, K. (2010). Route preferences among adults in the near market for bicycling: Findings of the cycling in cities study. American Journal of Health Promotion, 25(1), 40-70. https://doi.org/10.4278/ajhp.081006-QUAN-236

Winters, M., Teschke, K., Grant, M., Setton, E. \& Brauer, M. (2010). How far out of the way will we travel? Built environment influences on route selection for bicycle and car travel. Transportation Research Record: Journal of the Transportation Research Board, 2190. http://dx.doi.org/10.3141/2190-01

Yang, C. \& Mesbah, M. (2013, October). Route choice behaviour of cyclists by stated preference and revealed preference. Australasian Transport Research Forum (ATRF), Brisbane, Australia. Em http://atrf.info/papers/2013/2013_yang_mesbah.pdf

Zaiontz, C. (2017). Real statistics using Excel. Real Statistics Resource Pack. Em http://www.realstatistics.com/free-download/real-statistics-resource-pack/

Zhao, P. (2014). Theimpact of thebuiltenvironmenton bicyclecommuting: evidencefrom Beijing. Urban Studies, 51(5), 1019-1037. https://doi.org/10.1177/0042098013494423 